\title{
Assessment of rehabilitation in patients with spinal cord injuries: methodological considerations
}

\author{
P Daverat MD
}

\section{Service de Rééducation Fonctionnelle Neurologique, Hôpital Pellegrin, 33076 Bordeaux Cédex, France.}

It is becoming a necessity to predict the outcome of spinal cord injured patients, because their prognosis has greatly improved in recent years. The assessment of rehabilitation should be done with methodology to avoid selection bias and confounding factors, and to choose criteria for measuring outcome. Some simple rules are proposed in order to correctly use major statistical models.

Keywords: spinal cord injury; rehabilitation; assessment; prognosis.

In recent years, the progress of medical care, especially rehabilitation management, has greatly improved the outcome for disabled people, who can very often live independently if the necessary support services are provided. For economic reasons as well as for developing new therapeutic strategies, it is becoming necessary to predict the outcome of a disability. The best illustration is that many studies have appeared on the topic in the past few years, especially in the field of spinal cord injuries (SCI). In patients who survive the acute stage, prediction of the functional outcome is of great importance, but remains difficult because of methodological problems including selection bias, timing of the initial assessment, criteria for measuring outcome and the role of confounding factors. Our objective is to simply clarify some methodological points that should be included in every prognostic study, with specific reference to SCI.

One prerequisite in every prognostic study is the systematic inclusion of all patients with the condition under consideration, and, more important, the systematic statement of the inclusion criteria - if these are of sufficient scope to include most of the patients of interest. For SCI patients, selection bias could be especially harmful if secondary admissions to a specialised ward were included, because this would mean the prior exclusion of all patients dying during their primary management. To minimise such bias, it is necessary to include consecu- tive patients at the onset of their disability. The problem is easy to solve in the case of injuries critical events (ie stroke), but it is almost impossible in patients with chronic diseases to define the ideal timing for the initial assessment. The research protocol must be defined as a function of time. A prognostic study should be prospective, that is relative to future patients. Retrospective studies are not satisfactory, because, for example, they do not take into account patients who were lost during the follow up period. One other prerequisite is to define precisely the follow up period. Some studies have used the degree of improvement in functional status as the outcome measure; others have used the functional status at discharge or at set times after injury. Because predictors for each of these outcomes may differ, comparisons between various studies are difficult. Since the length of hospital stay varies enormously both among hospitals and among patients in a given hospital, the measurement of functional status at discharge can result in faulty conclusions. To avoid these difficulties, it seems better to choose a target date of assessment after the onset of the study (for example, 6 months, 1 year or 10 years are the most common follow up periods).

The first methodological point is to correctly state the problem that is to be solved, that is, to study the evolution of the sickness with four objectives. The first is a description; the second is a prediction - to estimate the mean evolution of the sickness; the third 
is comprehension - to study the relations between prognostic factors and evolution; and the fourth is medical decision making to propose a predictive model of the evolution to the physicians.

The second point is the choice of the dependent variable for prediction. It is very important to clearly define its properties. The dependent variable could be qualitative (eg survival), quantitative (eg Yale Scale Score $^{1}$ ) or ordinal (eg Frankel classification). ${ }^{2}$ Whilst staying clinically meaningful judgement criteria should be quantitative in order to be useful and valid for mathematical models. It is much more difficult to use ordinal variables.

The third point is the selection of potential prognostic factors that could be predictive of evolution. Some are probably related to the evolution (eg age, neurological level in SCI); others are possibly related to the evolution (eg initial vigilance, vegetative troubles in SCI); and others are a priori independent of the evolution (eg sex in SCI). We have to take into account the two first factors, but not necessarly the third. For these reasons, an exhaustive review of the literature must be done before beginning the analysis, in order to make an appropriate selection.

The fourth point is the statistical analysis. Before predicting the evolution, we have to describe it, that is to estimate the probability of the event as a function of time. Simple statistical models can be used, such as the Kaplan-Meier analysis or actuarial analysis. ${ }^{3}$ Patients who died or were lost during the follow up period should be included in the model.

The prediction of the evolution as a function of the selected factors can be made by univariate analysis. This type of analysis measures the relationship between one selected factor and the dependent variable. For example, in a previous study ${ }^{4}$ we have compared the recovery of walking in SCI between tetraplegics and paraplegics: $24 \%$ of the tetraplegics and $38 \%$ of the paraplegics were functional walkers at one year. But this apparent difference was not significant in this study, in which 157 patients were included $(p=0.14)$. Another example of univariate analysis studied the recovery of walking as a function of age. Patients who were over 60 years old were better walkers at one year than were those who were under 20 , but this difference was not significant $(p=0.55)$. This rather surprising result led us to further compare the initial YSS as a function of age. YSS is the Yale Scale Score,${ }^{1}$ from 0 to 10 , that allows grading of the sublesional neurological effects of a spinal cord injury. 0 indicates a complete lesion and 10 stands for normal spinal function. Thus the lower the YSS, the more severe is the lesion. Sixty-seven percent of patients under 20 were complete, only $31 \%$ over 60 were complete. The mean YSS was lower among young people (1.5) than among older people (3). This explains why age was not a predictive factor of the walking status and focuses on the limitation of the univariate analysis.

The multivariate analysis, that is with multiple prognostic variables, allows a correlation between the dependent variable with a combination of several factors. For example, we used the Cox model ${ }^{5,6}$ to correlate the recovery of walking in patients with SCI with a combination of three factors: age, level of injury and YSS. Age, which was not significant with univariate analysis, now becomes significant ( $p=$ 0.02 ). This method of adjustment avoids confusion biases, as the youngest people have the most severe injuries. But a new problem arises, that of 'multicolinearity'. In a previous study ${ }^{7}$ we found three independent predictors of survival in SCI: age, initial conscious level and respiratory assistance. These results were not in agreement with previous series which found the most important predictors to be age, lesion level and severity of the neurological injury. However, these studies have used univariate analysis in their data set, and a confounding bias was likely to be present. Lesion level was not found to be an independent predictor of survival, because it is related to respiratory assistance which is in itself a more important predictor. In fact, respiratory assistance is a clinical decision, and thus can really be used as a variable for some conditions when necessary. When two variables are colinear, ie vary together in a parallel fashion (in the same direction and 
with similar magnitudes) the same effect could be explained by either variable, which results in a reduction of power when analysing the contribution of each. Thus, when shifting from univariate to multivariate analysis (as in multiple regression models or Cox model), the apparent infuence of such multicolinear test variables can be greatly reduced or even disappear. To prevent multicolinearity between some obviously related test variables, a tree structured logistic (TSL) analysis can be used. ${ }^{8}$ This strategy involves grouping related test variables into factors, which are then analysed before the individual test variables. The advantage over more classical procedures (ie a step-down procedure) is that TSL analysis does not eliminate independent contribution of each related test variable in a factor.

The last stage of statistical analysis is to propose a predictive model of the evolution. On the basis of the significant factors derived from multivariate analysis, the model represents a medical decision making strategy.

Some simple methodological rules are now necessary in rehabilitation in order to correctly use powerful statistical models. From an epidemiological point of view, this is the only way to accurately estimate the prognosis and to precisely evaluate new procedures (therapeutic assays or technical aids).

\section{References}

1 Chehrazi B, Wagner F, Collins W, Freeman D (1981) A scale for evaluation of spinal cord injury. $J$ Neurosurg 54: 310-315.

2 Frankel HL, Hancock DO, Hyslop G et al (1969) The value of postural reduction in the initial management of closed injuries of the spine with paraplegia and tetraplegia. Paraplegia 14: 262-275.

3 Kaplan EL, Meier P (1958) Non parametric estimation from incomplete observations. J Am Stat Assoc 53: 457-481.

4 Daverat P, Sibrac MC, Dartigues JF, Mazaux JM, Marit E, Debelleix X, et al (1988) Early prognostic factors for walking in spinal cord injuries. Paraplegia 26: 255-261.

5 Cox DR (1970) In: The Analysis of Binary Data. Chapman and Hall, London.

6 Cox DR (1972) Regression models of life tables. J Statis Soc 34: 187-220.

7 Daverat P, Gagnon M, Dartigues JF, Mazaux JM, Barat M (1989) Initial factors predicting survival in patients with a spinal cord injury. J Neurol Neurosurg Psychiatry 52: 403-406.

8 Commenges D, Dartigues JF, Peytour P, Puymirat E, Henry P, Gagnon M (1989) A strategy for analysing multiple risk factors with application to cervical pain syndrome. Methods Inf Med 28: 14-19. 